\title{
Mallas tensegríticas de doble capa y manipulaciones de Rot-Umbela
}

\section{Double-layer tensegrity grids and Rot-Umbela manipulations}

\author{
V. Gómez-Jáuregui ${ }^{*}$
}

\section{RESUMEN}

Las mallas tensegríticas de doble capa (MTDC) son estructuras de Tensegridad que contiene dos mallas traccionadas paralelas, unidas por otra capa intermedia compuesta por elementos comprimidos y traccionados verticales y/o diagonales.

Este trabajo presenta inicialmente un breve repaso sobre las propuestas de MTDC que se han realizado hasta el momento, exponiendo luego una nueva metodología, eminentemente geométrica, para la generación de este tipo de estructuras tensegríticas.

Tras aplicar la propuesta de Otero para generar mallas de doble capa (MDC) convencionales, se procede a realizar Manipulaciones de Rot-Umbela en las capas inferior $y / o$ superior para convertir dicha estructura en una MTDC. Las Manipulaciones de Rot-Umbela consisten en la apertura de cada vértice en un cierto polígono, al cual se le aplica una rotación o giro particular. Se consigue así un potente sistema para "tensegritizar" MDC convencionales y abrir una línea de investigación muy interesante en el campo de la Tensegridad.

458-9

Palabras clave: Tensegridad; estructuras; mallas; doble capa; Rot-Umbela.

\section{SUMMARY}

Double-layer tensegrity grids (DLTGs) are tensegrity spatial systems containing two parallel networks of members in tension forming the top and bottom chords, whose nodes are linked by vertical and/or inclined web members under compression and tension.

This paper presents, as an introduction, a brief perspective of the historical proposals for DLTGs over the last years, describing later a new approach for generating these kinds of structures, mainly in geometrical terms.

After applying Otero's proposal for designing conventional double-layer grids (DLGs), a new technique, known as Rot-Umbela Manipulation, is applied to their upper and/or lower layers for generating DLTGs. Rot-Umbela Manipulation consists of opening a vertex in the plane for obtaining a certain polygon, which is then rotated by a determined angle. This powerful operation opens an endless catalogue of DLTGs and a very interesting line of research in the field of Tensegrity.

Keywords: Tensegrity; structures; grids; double-layer; Rot-Umbela.

\footnotetext{
(") Universidad de Cantabria. Santander (España). 
1. Flat Out. Escultura tensegrítica de Kenneth Snelson (1979).

2. Bocetos realizados por Kenneth Martin.
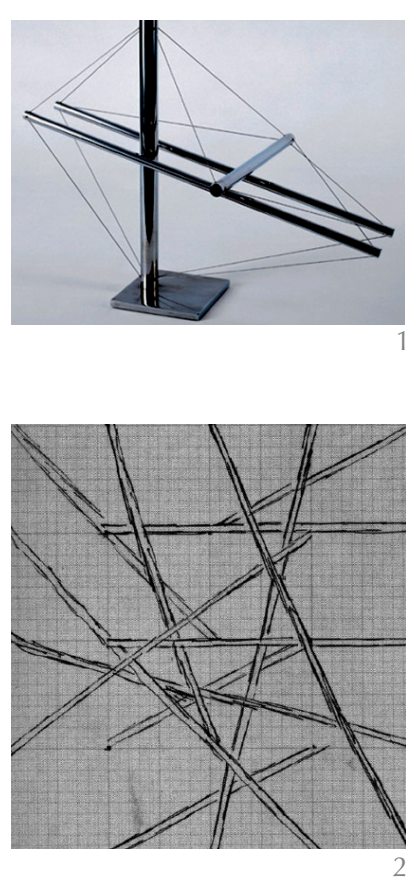

\section{INTRODUCCIÓN}

Las estructuras tensegríticas son unas grandes desconocidas en el campo de la ingeniería y la arquitectura, aunque en los últimos años, gracias a las numerosas investigaciones que se están realizando en ciertos centros tecnológicos, se están abriendo camino paulatinamente. La Tensegridad, pese a no tener una definición universalmente aceptada, puede ser considerada como un principio estructural basado en el empleo de componentes aislados comprimidos que se encuentran dentro de una red traccionada continua, de tal modo que los miembros en compresión (generalmente barras) no se tocan entre sí y están unidos únicamente por medio de elementos traccionados (habitualmente cables) que son los que delimitan espacialmente dicho sistema, que está en equilibrio y es estable por sí mismo (1).

En otras palabras, las tensegridades son estructuras desnudas que muestran inequívocamente los esfuerzos que sufren sus elementos. Una ilustración sencilla de dicho principio puede verse en la escultura de Kenneth Snelson de la Figura 1, en donde se puede apreciar que los cables están sometidos única y exclusivamente a tracción, mientras que los tubos trabajan específicamente a compresión.

Qué duda cabe que, independientemente de las posibilidades que estas estructuras pueden ofrecer en ámbitos más prácticos, existe una componente formal o plástica que supone un gran atractivo para cualquier sistema que comulgue con estos principios. Como ejemplo paralelo, a modo de representación de la estética propia de estos elementos lineales colocados arbitrariamente en el espacio, se podrían mencionar algunos de los trabajos de Kenneth Martin (2), en los que el caos lógico y sistemático originado por la interpretación del orden de un determinado entramado genera esquemas como el de la Figura 2. Es curioso el parecido relativo que tiene con las configuraciones tensegríticas.

En su libro "Godel, Escher, Bach-An Eternal Golden Braid", Douglas Hofstadter incluye una frase relativa a la Teoría de Números, afirmando que es la más pura de las ramas de las matemáticas por ser la única que no tiene aplicaciones. Del mismo modo, también se podría decir que la Tensegridad es la más pura de las tipologías estructurales por no contar tampoco con ninguna aplicación relevante. Siguiendo con la analogía, se podría considerar que los números enteros, objeto de estudio de la Teoría de Números, son elementos aislados, separados por infinitos números fraccionales e irracionales, y relacionados únicamente por medio de tirantes leyes aritméticas. Sin embargo, como se está demostrando en los últimos años, ambos campos de la ciencia están contando con utilidades en número creciente.

La compresión flotante, como también se denomina a la Tensegridad, está siendo considerada cada vez más frecuentemente por parte de arquitectos e ingenieros para la construcción de cubiertas, pasarelas e incluso puentes (como el Kurilpa Bridge, en Brisbrane, Australia). Paradójicamente, cualquier estructura compuesta por cables y montantes empieza a ser considerada tensegrítica por algunos profesionales (legos en la materia), lo cual induce a error; pero no cabe duda de que hay una creciente sensibilidad en la aplicación de estas estructuras en el ámbito de la construcción. A este respecto, es importante aclarar que no se pueden considerar como tensegridades "puras" a todas aquellas estructuras que no son auto-estables y no pueden mantenerse en equilibrio por sí mismas; en otras palabras, estructuras que necesitan un elemento exterior arriostrante, un anclaje externo o una cimentación que le dé soporte. Es el caso de, por ejemplo, las grandes cubiertas de cables radiales o de rueda de bicicleta (como la Georgia Dome en Atlanta, la más grande del mundo de este tipo), que se anclan en todo su perímetro en un anillo de compresión de hormigón armado. De este modo, dejan de cumplir una de las condiciones de la definición aportada inicialmente, la de que han de ser los elementos traccionados (bien cables, bien membranas) los que delimiten espacialmente el sistema.

Basándonos en la definición de Tensegridad aportada inicialmente, podemos definir las Mallas Tensegríticas de Doble Capa (MTDC) como un sistema espacial tensegrítico consistente en dos mallas traccionadas paralelas, una superior y una inferior, unidas entre sí por otra capa intermedia compuesta por elementos verticales y/o diagonales (barras comprimidas y/o cables traccionados). Para comprender este concepto más fácilmente, en este artículo se revisarán las propuestas que a lo largo de las últimas décadas se han ido realizando en lo concerniente a MTDC, desde el origen hasta nuestros días, para de este modo poder analizar los dos métodos básicos (por composición y descomposición) que se han venido empleando para la generación de estas mallas.

A continuación, se introducirán dos conceptos importantes. Por una parte, se explicará la técnica aportada por Otero para la generación de Mallas de Doble Capa (MDC) convencionales, también denominadas simplemente estructuras espaciales; 
y por otra parte, se definirá brevemente lo que suponen las manipulaciones por Umbela. A partir de este punto, ya estarán establecidas las bases para presentar una nueva propuesta para la generación de MTDC a partir de MDC convencionales: las Manipulaciones de Rot-Umbela. Se comprobará que algunas de las mallas tensegríticas más comunes se pueden obtener mediante este método y se mostrarán algunos ejemplos nuevos que ilustren la potencia y utilidad de esta nueva metodología.

Finalmente, se apuntarán brevemente algunas posibles aplicaciones de las MTDC, se expondrán propuestas futuras y se explicarán las conclusiones principales obtenidas tras este análisis, que supone tan solo una parte del completo estudio al que se están sometiendo este tipo de estructuras.

\section{CONTEXTO HISTÓRICO}

Desde el controvertido descubrimiento de la Tensegridad (3) a finales de la década de los 40, numerosas configuraciones de maIlas tensegríticas se han propuesto por parte de diversos investigadores. Durante esos primeros años y comenzando con sus propias patentes, fueron principalmente Fuller (4), Snelson (5) y Emmerich (6) (7) los que empezaron a diseñar y construir modelos y maquetas de estas tensegridades planas, esencialmente diferentes de las más tradicionales estructuras esféricas, prismáticas o longitudinales. Algunos de esos diseños (8) (9) no fueron mostrados públicamente hasta fechas recientes, pese a que podrían haber resultado de indudable interés para su análisis y estudio.

Ya en los años 70, Pugh (10) de manera predominante, y también Vilnay (11) propusieron otros tipos diferentes de mallas de Tensegridad desde un punto de vista eminentemente geométrico. No obstante, no sería hasta la siguiente década cuando Hanaor (12) (13) (14) (15) (16) (17) (18) y Motro (19) (20) (21) (22) (23) (24) tomaron una perspectiva más estructural y analítica, profundizando en otros aspectos como la búsqueda de forma, resistencia, estabilidad, deformación, etc. El primero de ellos, Ariel Hanaor, experimentó de modo más intensivo con la yuxtaposición de prismas tensegríticos (para mallas planas) o pirámides truncadas (para configuraciones abovedadas) evitando en lo posible el contacto entre barras de modo similar a como lo hiciera Emmerich (tensegridades de clase 1 , por confluir en cada nudo tan sólo un elemento a compresión). Por su parte, René Motro estudió mallas compuestas por pirámides tensegríticas truncadas de cuatro barras, también denominados "ten- segrity quadruplex" (ver Figura 3a, esencialmente los mismos semicuboctaedros mostrados por Emmerich (6) en su primera patente, tal y como muestra la Figura 3b), pero anexionándolos de tal forma que los extremos de sus barras se tocaran entre sí (obteniendo tensegridades de clase 2 y 4 ). Paralelamente, Nestorovic (25) (26) desarrollaba sus propias propuestas para lo que él denominaría cúpulas de integridad tensional.
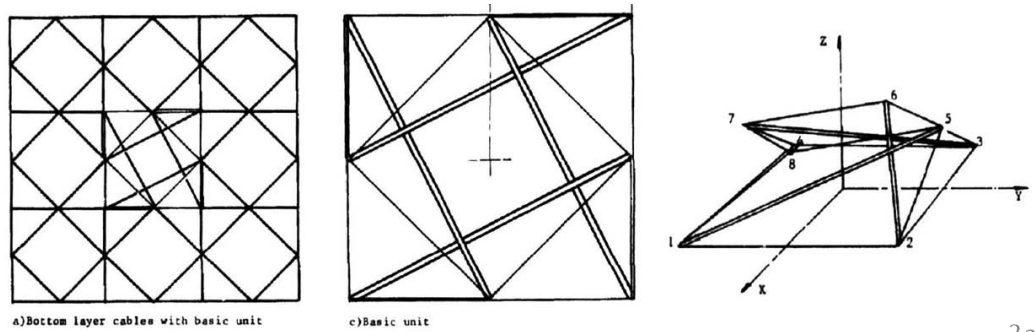

3. Mallas de "quadruplex" piramidal o semicuboctaedros tensey por Emmerich (b). gríticos propuestos por Motro (a)

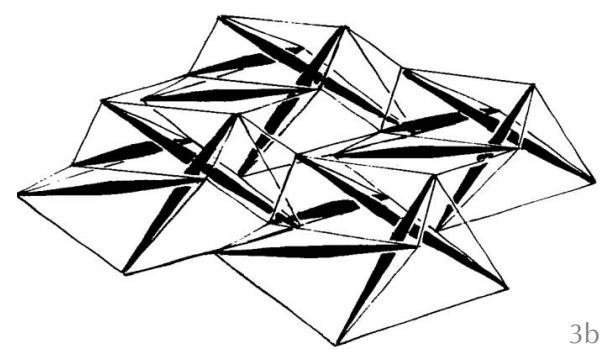

Durante esa misma época, más concretamente en 1988, Emmerich (27) (28) (29) publicaba un completo y exhaustivo compendio de teorías, estructuras, modelos y proyectos llevados a cabo por él mismo y por sus estudiantes. Uno de los capítulos de su libro está dedicado exclusivamente a lo que él denominaba "redes planas autotensadas", aunque de manera restrictiva, pues solo tendría en cuenta las MTDC compuestas por yuxtaposición de módulos estables. No obstante, consiguió aglutinar numerosísimas mallas compuestas por módulos prismáticos, piramidales, antiprismáticos, antipiramidales, entrelazados e interpenetrados, generando diversas y variadas estructuras espaciales de doble capa.

Siguiendo este ejemplo, a finales del siglo pasado, otros estudios se han Ilevado a cabo por parte de diversos autores, que aunque no hayan modificado en esencia el modo de componer nuevos sistemas, sí que han contribuido de modo significativo a analizar y calcular el comportamiento de estas estructuras. Uno de los más prolíficos sería Wang B.B. (30) (31) (32) (33) (34) (35) (36) (37) (38), quien desde 1996 se dedicó a estudiar de manera rigurosa diferentes construcciones tensegríticas modulares, y más concretamente MTDC. En particular, diferenció dichas estructuras según fuera su módulo básico, la yuxtaposición y tipo 
4. Mallas Tensegríticas de Doble Capa de Emmerich (a), Snelson (b) y Kono\&Kunieda (c) basadas en trípodes tensegríticos.

5. Patente de Raducanu y Motro: a) MTDC formada por separadores V22, b) Detalle de separadores $\mathrm{v} 22$. de unión entre ellos, la conexión o aislamiento entre elementos a compresión, la rigidez o flexibilidad del conjunto, la ubicación y tipo de los apoyos, etc. En otras palabras, continuó la labor que iniciara Emmerich pero desde un punto de vista más estructural que geométrico. En la misma línea, Hilyard and Lalvani (39) se dedicaron a la creación de otros entramados bidimensionales tomando como punto de partida el modo de cubrir el plano mediante células espaciales, las cuales bautizarían como "estructuras tipo Emmerich", pues no eran otra cosa que módulos prismáticos tensegríticos.

Paralelamente, Kono y Kunieda (40) (41) (42) (43) (44) (45) experimentaron con otro tipo de mallas de compresión flotante, caracterizadas por su sencillez y elegancia, y basadas en el empleo de trípodes interconectados o, lo que es parecido, pirámides truncadas tensegríticas, de tres barras cada una. Curiosamente, y como se puede apreciar en la Figura 4, eran muy similares al diseño $\mathrm{n}^{\circ} 4$ de la patente de Emmerich (6) (aunque ésta tenía algunos vértices conectados directamente) así como a la patente abandonada de Snelson (8). Durante su proyecto, probaron diferentes geometrías, que dependían fundamentalmente de cómo estuvieran conectados los cables de la base mayor de dichos módulos: bien directamente a las barras, bien al ortocentro de éstas o bien a algún punto intermedio de sus bisectrices. Como resultado, construyeron un prototipo a gran escala, compuesto por 33 trípodes modulares que cubrían una superficie de $80 \mathrm{~m}^{2}$, empleando además un diseño novedoso para el sistema de enlace en cada uno de los nudos. Así mismo, todo el trabajo se vio reflejado en la concesión de una patente (45) que incluía también el diseño de MTDC abovedadas y de mallas compuestas por prismas de 4 barras.

Por su parte, aunque trabajando en la misma línea de conseguir dar aplicación a las MTDC, el Laboratorio de Mecánica e Ingeniería Civil (LMGC) de la Universidad de Montpellier, liderada por René Motro, ha estado albergando, dirigiendo y supervisando desde 1997 a numerosos estudiantes de doctorado, máster y grado dedicados a esta misma temática: Quirant (46) (47) (48), Smaili (49) (50) (51) (52), Sánchez (53) (54), Bouderbala (55), Vassart (56), Averseng (57) (58) (59) (60), Djouadi (61), Kebiche (62), Le Saux (63), Raducanu (64), etc. Sus tesis y proyectos han estado enfocados a muy diversos aspectos, como metodologías de búsqueda de forma (form-finding), estados de pretensado, configuraciones desplegables, técnicas constructivas, control activo del sistema, dimensionamiento óptimo, etc. Aun y con todo, la mayoría de esos trabajos han estado aplicados a, básicamente, tan sólo dos configuraciones de mallas: la primera es la misma composición de semicuboctaedros tensegríticos que estudiara Motro en los años 80 (Figura 3b); y la segunda está compuesta por varias alineaciones, entrelazadas y perpendiculares entre sí, con las barras en zigzag (Figura 5a) o, lo que es lo mismo, formadas por separadores en forma de $\mathrm{V}$ en dos direcciones, que por lo tanto denominaron V22 Expanders (Figura 5b). Se puede apreciar una curiosa similitud con algunas de las mallas planas que construyera Snelson en los años 60 (Figura 6).
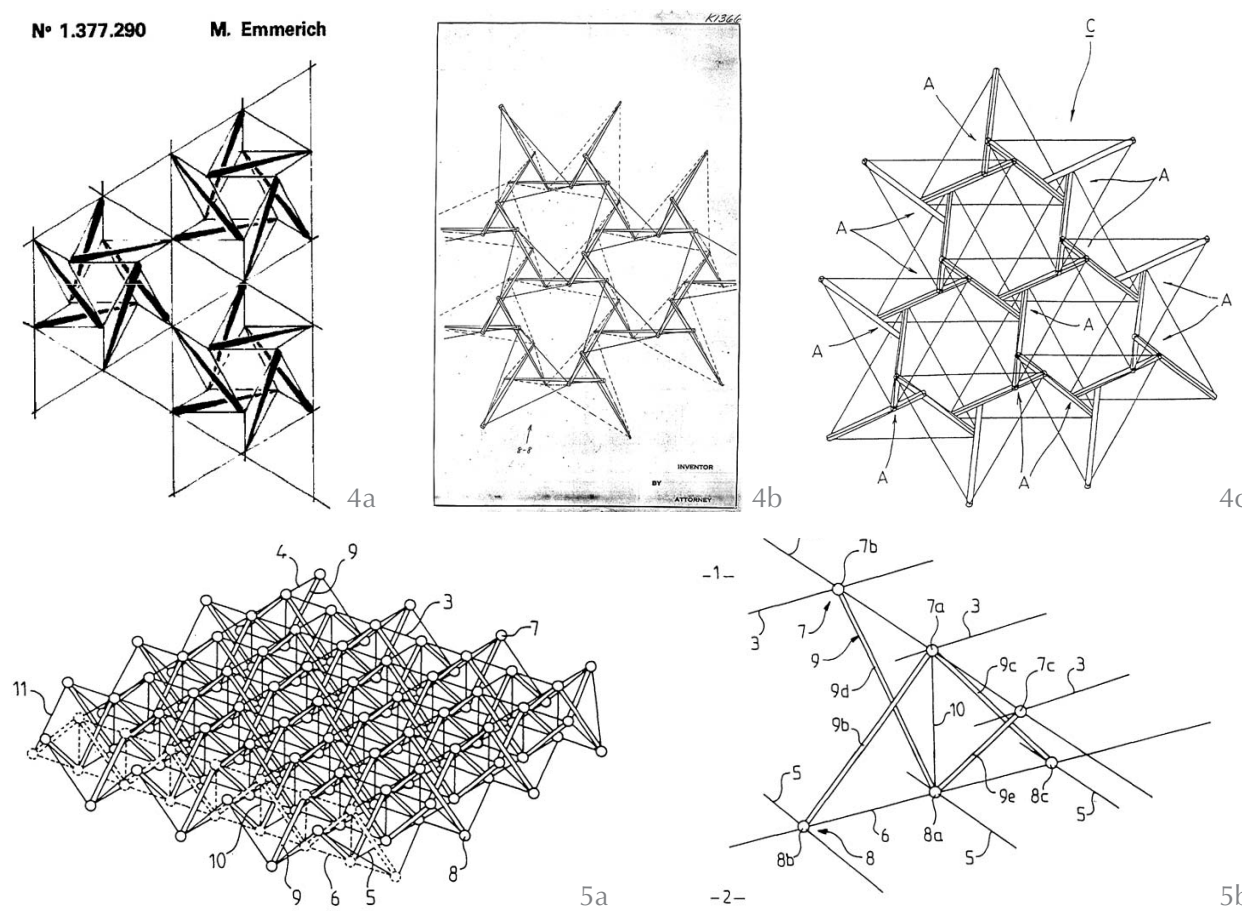
Destaca entre todos esos trabajos de investigación, por su originalidad y potencial, la tesis doctoral escrita por Raducanu (64) en el año 2001, y que tomó parte importante del proyecto Tensarch que se estaba llevando a cabo en dicho laboratorio de Montpellier. El autor analizó los sistemas tensegríticos y las MTDC existentes hasta entonces para, desde ese punto de partida, realizar sus propias propuestas de modo original y sistemático. Su aportación esencial fue la incorporación de separadores característicos (en forma de V, Y o Z) entre las dos redes de cables tensados superior e inferior, asegurando adicionalmente la estabilidad del conjunto mediante conexiones adecuadas en el contorno de las mallas. Esta innovadora técnica propició la generación de 15 nuevas configuraciones de mallas, no todas ellas de doble capa propiamente entendidas, y lo que es más importante, un nuevo método que no estaba basado en la yuxtaposición de módulos ya estables de por sí, sino de elementos dependientes unidos mediante relaciones geométricas y topológicas. Durante dicho proceso de estudio, se construyeron y testaron diferentes prototipos a gran escala, y como resultado se desarrolló una patente (extracto en Figura 5) mostrando las innovaciones esenciales originadas a partir de esta línea de investigación.

Además de la realización de estos estudios eminentemente teóricos, también ha habido interesantes realizaciones prácticas, como el proyecto de Passera y Pedretti's (65) para la Expo de Suiza de 2002, con la construcción del Blur Building (Figura 7), un pabellón que emanaba vapor de agua para convertirse en una "nube habitable" sobre un lago. Pero como ocurre también con las cúpulas diseñadas por Addriansens y Barnes (66), este tipo de estructuras no son propiamente consideradas MTDC por ser composiciones de octaedros tensegríticos como los que ya expusiera Pugh (10).
En todo caso, es interesante reseñar que hoy en día no ha decrecido en absoluto el interés por las MTDC; tal es así que diversos equipos de investigación, como Panigrahi, Gupta y Bhalla (67) (68), han estado construyendo y poniendo en carga para su análisis estructural prototipos de mallas desmontables para su aplicación en cubiertas desplegables. También sirve el ejemplo de Tran y Lee (69) (70) (71), que han seguido investigando diversos aspectos relacionados con la búsqueda de forma y los estados iniciales de pretensado de los dos tipos de mallas más comunes hasta el momento. O finalmente, las labores de Liapi y Kim (72), que continúan indagando en nuevas aplicaciones arquitectónicas para las mallas de compresión flotante.

\section{METODOLOGÍAS DE DISEÑO DE MTDC}

Como corolario de las experiencias enumeradas en el apartado anterior, se puede deducir que son dos los métodos básicos para diseñar MTDC que se han venido empleando hasta el momento, y que a continuación resumiremos:

\subsection{Composición}

La creación de mallas se realiza mediante la unión de varios módulos tensegríticos, estables por sí mismos. Las células básicas consideradas son habitualmente prismas o pirámides truncadas tensegríticas, de simetría cilíndrica o axial, constituidas generalmente por 3 o 4 barras ("simplex" o "quadruplex" de las Figura 8 y 9). No obstante, también existen otras configuraciones dignas de análisis, como las planteadas por Emmerich (27), que podrían reabrir una fructífera línea de investigación.

Dependiendo de los tipos de conexiones entre módulos, los sistemas así generados se pueden clasificar como sigue:

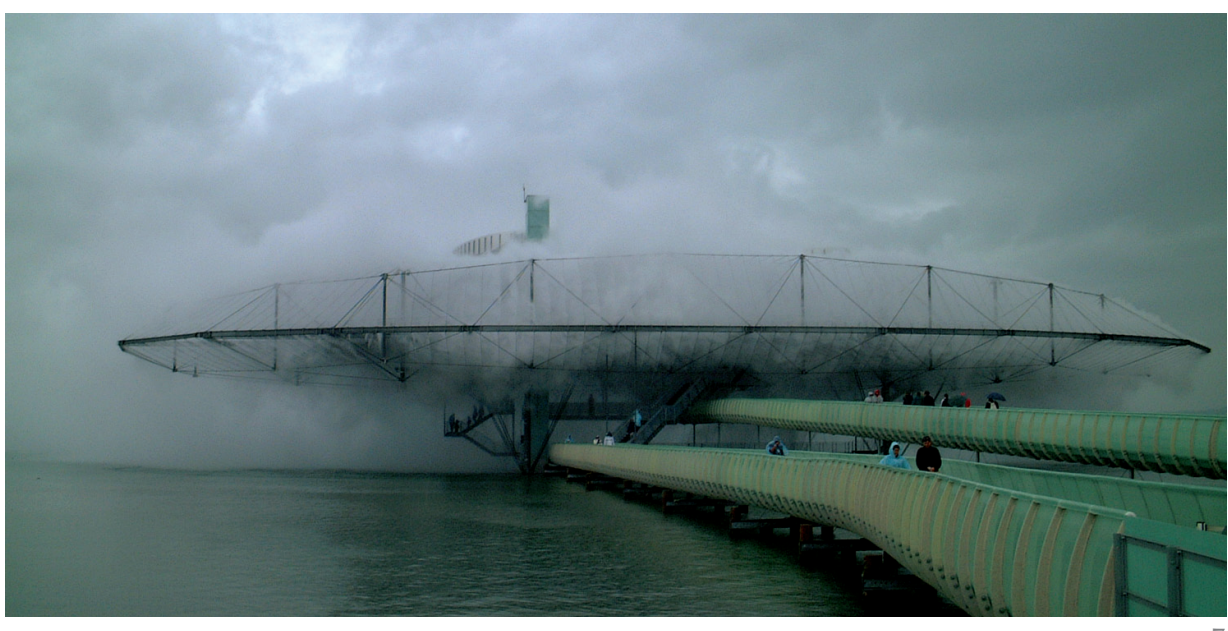

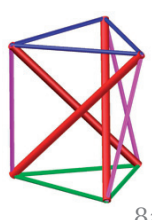

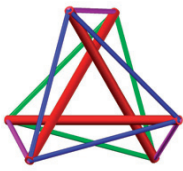

$8 a$

$8 \mathrm{~b}$
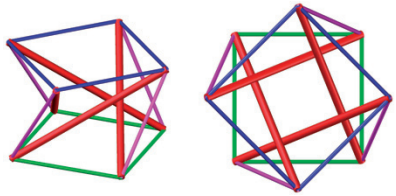

96 
10. Tipos de conexiones. Con barras aisladas: a) Vértice-Arista Unilateral, b) Vértice-Arista Bilateral, c) Arista-Arista, d) Con barras contiguas.

11. a) Separador V33, b) Separador V44, c) Separador Z6, según Raducanu (64).

\subsubsection{Barras Aisladas $(k=1)$}

Los elementos comprimidos no se tocan entre sí, estando unidos únicamente mediante elementos traccionados. Se dan las siguientes variantes básicas:

\subsubsection{Conexión Vértice-Arista}

\subsection{Unilateral}

(Tipo la (12) o topología A (73)): dos vértices de ambas capas superior e inferior de un mismo módulo intersecan dos aristas, superior e inferior respectivamente, de otro módulo (Figura 10a).

\subsection{Bilateral}

(Tipo lb (12) o topología B (73)): el vértice de un módulo interseca la arista de otro en una de las capas (p. ej. superior) mientras que a su vez una arista suya es contactada por un vértice del otro módulo en la otra capa (p.ej. inferior) (Figura 10b).

\subsubsection{Conexión Arista-Arista}

Dos módulos adyacentes comparten la misma arista parcialmente (los vértices no coinciden) en ambas capas (superior e inferior). Tipo Il según Hanaor (12) (Figura 10c).

\subsubsection{Barras Contiguas $(k>1)$}

Los miembros en compresión de un módulo entran en contacto directo con los de módulos adyacentes por medio de sus vértices (Figura 10d). Mientras que algunos autores no consideran estos sistemas tensegridades puras, otros como Motro (22) reivindican su integridad tensegrítica puesto que se trata de un sistema continuo de cables dentro del cual hay un conjunto de componentes comprimidos aislados, considerando componente a un grupo de barras comprimidas y articuladas.
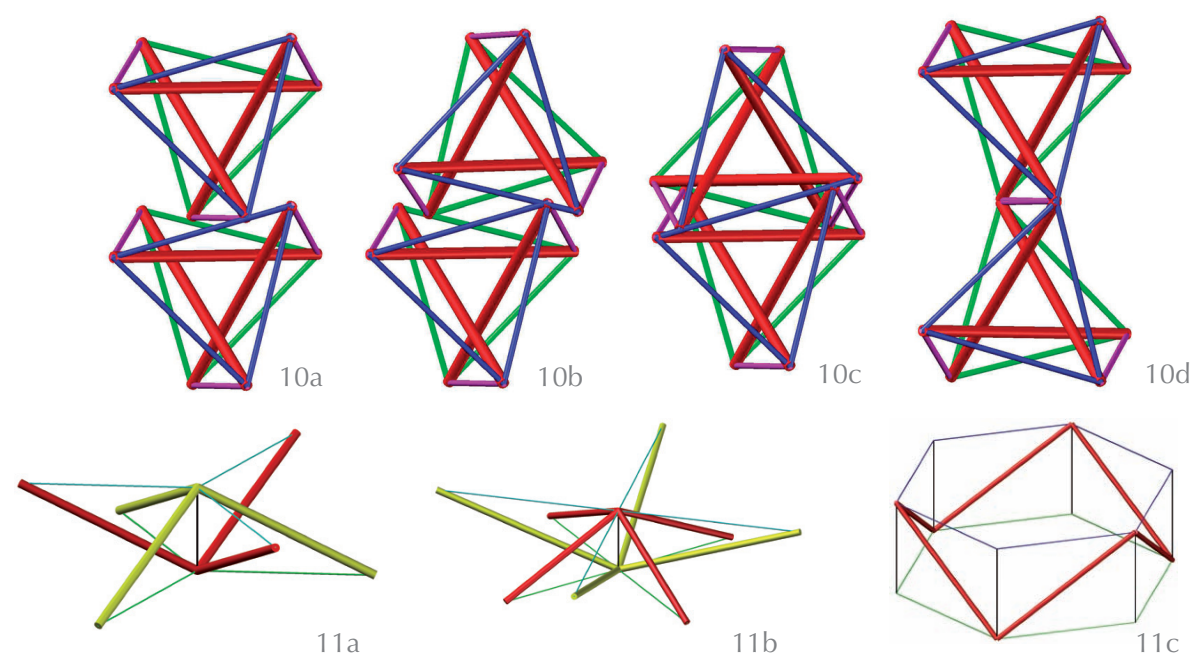
Finalmente, la tipología $Z n$ está formada por $n$ barras que, zigzagueando entre las dos capas, configuran un contorno poliédrico, como se puede ver en la Figura 11c.

Particularmente, durante la presente investigación se ha podido comprobar que, por ejemplo, la malla compuesta por los separadores V33, que se muestra en la Figura 12a, también puede ser generada a partir de la yuxtaposición de módulos estables como el de la Figura 12b. Dicho módulo está en equilibrio incluso sin la inclusión de uno de los tendones diagonales de la capa superior o inferior (aunque el estado de tensión y compresión de sus miembros se alteraría ostensiblemente), y no como aparece en la Figura 4 de la patente de Raducanu (sin ninguna de las dos diagonales) pues de ese modo colapsaría. Siempre y cuando cuente con ellos, la estructura será independientemente estable. Este hecho se puede confirmar en la Figura 13a con el módulo que permite generar la malla formada por separadores V44 (Figura 13b), en el cual la topología es exactamente igual (evidentemente, la geometría final difiere de la Figura $12 b$ debido a que las longitudes y tensiones de los elementos son diferentes).

\section{NUEVA METODOLOGÍA PARA OBTENER MTDC A PARTIR DE MDC CONVENCIONALES}

El principal objetivo de este artículo es el de presentar un nuevo método para "tensegritizar" mallas de doble capa (MDC) convencionales. El enfoque será eminentemente geométrico y conceptual, pues el proceso de búsqueda de forma definitivo y el proceso de cálculo de estados de tensión es subsecuente y dependiente de esta primera etapa.

Las MDC convencionales, comúnmente llamadas estructuras espaciales, son generalmente una composición de mosaicos regulares (triángulos equiláteros, cuadrados o hexágonos que rellenan el plano) ubicados en las capas superior e inferior, y en las que luego se conectan los vértices de ambos entramados mediante otra capa intermedia de conectores diagonales y/o verticales. Por lo tanto, ése es el proceso habitual, por ser el más intuitivo, para la obtención de dichas estructuras.

No obstante, Otero (75) (76) propuso un nuevo método para definirlas geométricamente, basado en el empleo de un mosaico que representara la capa intermedia de diagonales y, a partir de él y aplicando una serie de reglas, obtener el resto de nodos de la malla completa. Dichas normas son fundamentalmente dos, y rigen por una parte el modo de colocar los nodos en la capa superior y/o inferior basándose en la disposición de los vértices del mosaico diagonal, y por otra parte la manera de enlazar o unir dichos nodos en cada capa para así constituir los teselados inferior y superior que terminan de definir totalmente la MDC. Como resultado de aplicar este método, se consigue un sistema mucho más flexible y potente que permite partir de mosaicos diagonales no sólo regulares, sino también semirregulares, hemirregulares, equifaciales (duales de los semirregulares) y semiequifaciales (duales de los hemirregulares).

Actualmente se está llevando a cabo otra investigación colateral acerca de las numerosas posibilidades que arroja este planteamiento (77), pero que obviaremos por no ser ése el objeto del presente escrito.

Con el fin de ilustrar esta metodología, en la Figura 14 a se muestra el teselado semirregular 4, 6, 12, formado por cuadrados, hexágonos y dodecágonos. Para proceder con el método expuesto, consideraremos dicho mosaico como la capa de diagonales, para sobre él ubicar los vértices de la capa superior (círculos azules) alternados con los de la inferior (cuadrados verdes). Esta alternancia es tan sólo una de las opciones que plantea la ley de localización de nodos, lo mismo que podría haberse usado la de puntos medios de las aristas en una capa y todos los puntos de los vértices en la otra, etc. Del mismo modo y arbitrariamente, se define la norma de unión entre nodos de la misma capa, que será en este caso la de establecer conexiones entre los vértices más cercanos dentro del mismo polígono, generando las redes finas azules continuas en la capa superior y las verdes más gruesas y discontinuas en la inferior (Figura 14b). Como resultado, si trasladamos dicha disposición al espacio tridimensional, obtendríamos la MDC de la Figura 14c.

A partir de este punto, se podrían aplicar diferentes metodologías para convertir estas mallas rígidas en nuevas MTDC: Manipulación de Rot-Umbela (78), composición a partir de módulos estables de Emmerich, nuevos diseños intuitivos mediante sustitución de barras diagonales por cables, etc.
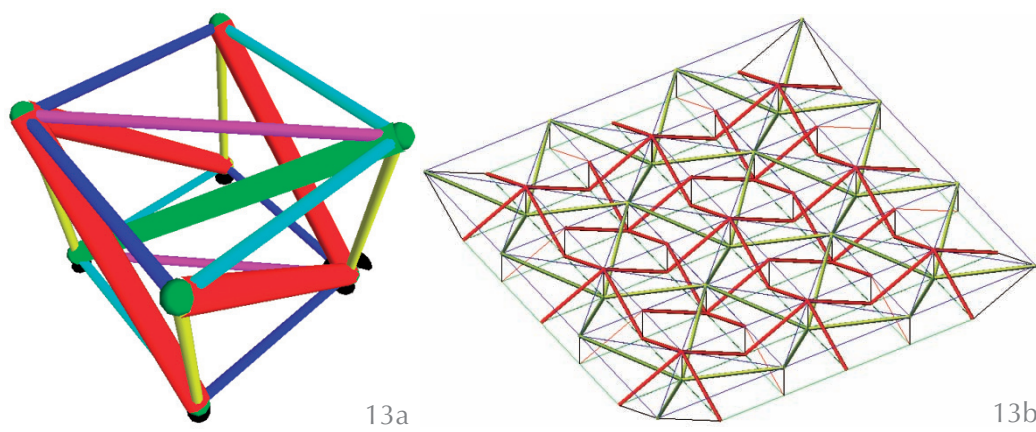

12. a) Malla compuesta por los separadores V33, b) Módulo estable que también compone dicha malla.

13. a) Módulo estable que también compone dicha malla, b) Malla compuesta por los separadores $\mathrm{V} 44$

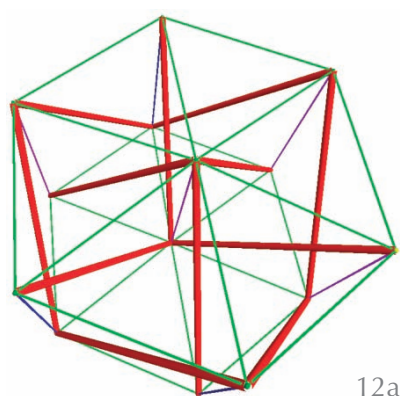

$12 \mathrm{a}$

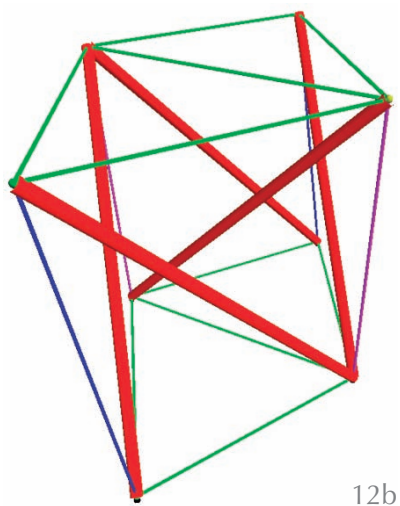

$12 b$ 
14. Generación de una MDC a partir del mosaico semirregular $4,6,12$.

15. Representación gráfica de una Manipulación en Umbela según Gancedo.

16. Manipulaciones de Rot-Umbela en un mosaico semirregular.
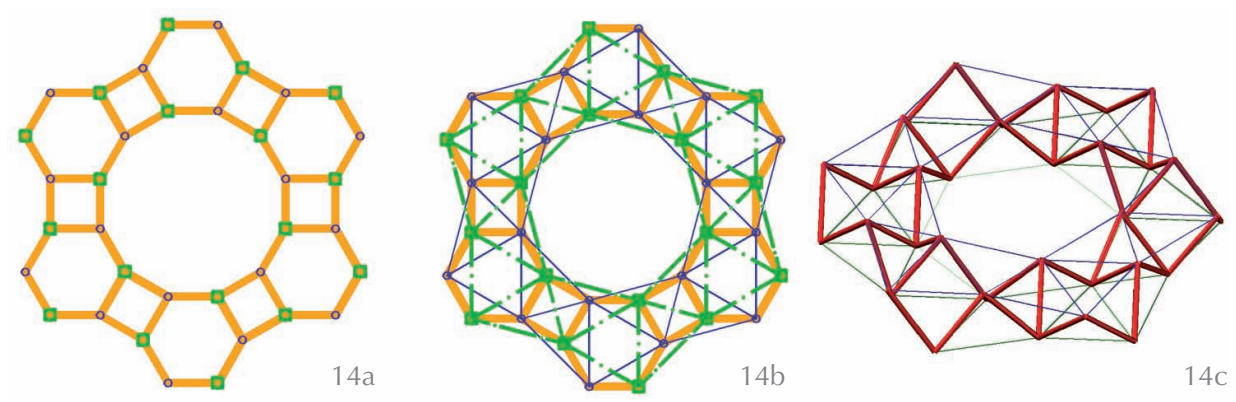

$14 \mathrm{C}$

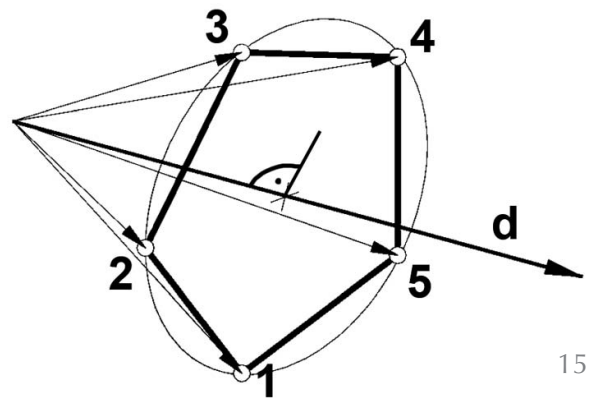

Sea como fuere, merece especial atención el tratamiento que se le dé a los elementos de borde, pues en buena medida serán estos los que aseguren la estabilidad final de la nueva malla tensegrítica y la correcta transmisión de esfuerzos a los apoyos.

\subsection{Manipulación de Rot-Umbela}

El presente concepto se deriva de otro ya existente propuesto por Gancedo (79) (80), a finales de los años 80, pero que tan sólo se había aplicado a los poliedros: se define Manipulación en Umbela a la operación que consiste en abrir una dirección $(d)$ dada en el espacio de tal modo que se obtenga un polígono regular con sus vértices ubicados en el plano perpendicular a dicha dirección (Figura 15).

Análogamente, aplicado a mosaicos y MDC, definiremos como Manipulación de Rot-Umbela a una manipulación en Umbela convencional en la cual la dirección dada $(d)$ es siempre perpendicular al plano en el que esté definida dicha red, pero con las salvedades de que los polígonos generados no tienen por qué ser regulares y que pueden girarse tomando como eje de rotación dicha dirección original $(d)$.
Considerando que un vértice tiene valencia $v$ cuando en él confluyen $v$ aristas del mismo plano, si tras sufrir una Manipulación de RotUmbela se convierte en un polígono de $u$ lados, su "valencia de umbela" será $u$. Si en un nodo concreto la valencia de vértice coincide con la valencia de umbela $(u=v)$, como sucede en los nudos A y B de la Figura 16, se dirá que tiene una "valencia de umbela natural". En dicha imagen se puede comprobar que el vértice $A$ consta de valencia 4 que se convierte en valencia de umbela 4, por lo que se trata de una valencia de umbela natural. Lo mismo ocurre con el vértice $B$, con la diferencia de que éste sufre así mismo una rotación de $120^{\circ}$ en torno al centro del nuevo polígono. Por último, el nodo C, de valencia 4, sufre una Rot-Umbela de valencia 3 , por lo que se genera un triángulo en vez de un cuadrilátero como en los otros casos.

En lo concerniente a las MDC, para obtener mallas tensegríticas a partir de éstas, se toma la consideración de que la capa intermedia está compuesta por barras a compresión mientras que las capas inferior y superior son redes tensadas compuestas por elementos a tracción. Desde esta configuración, la Manipulación de Rot-Umbela puede ser ejecutada tanto a una capa como a ambas, del mismo modo que se puede aplicar a todos los vértices o sólo a una fracción determinada de ellos. De modo natural se han de añadir conexiones adicionales entre los nuevos nodos así originados, que serán por norma general enlazados mediante nuevos tendones traccionados. Consecuentemente, las combinaciones y por tanto los resultados obtenidos en forma de MTDC son numerosísimos, dependiendo directamente de la complejidad de la MDC a la que se aplique.
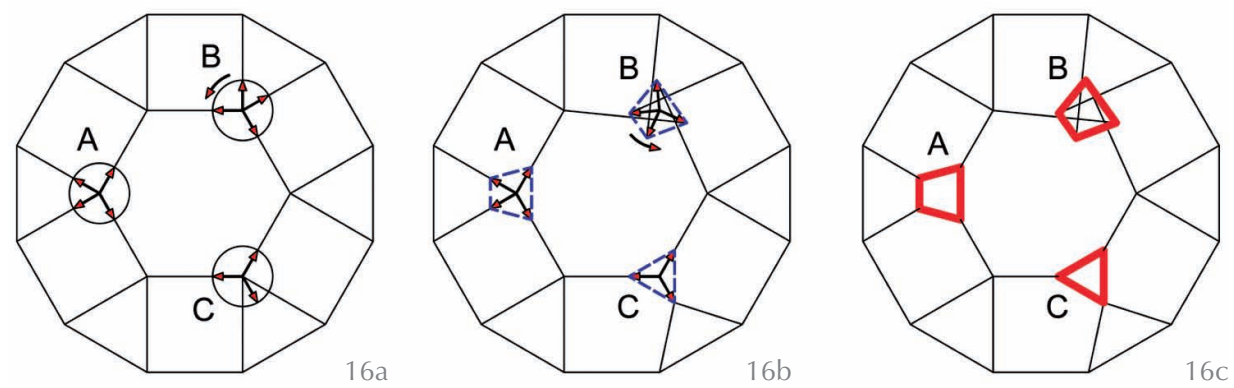


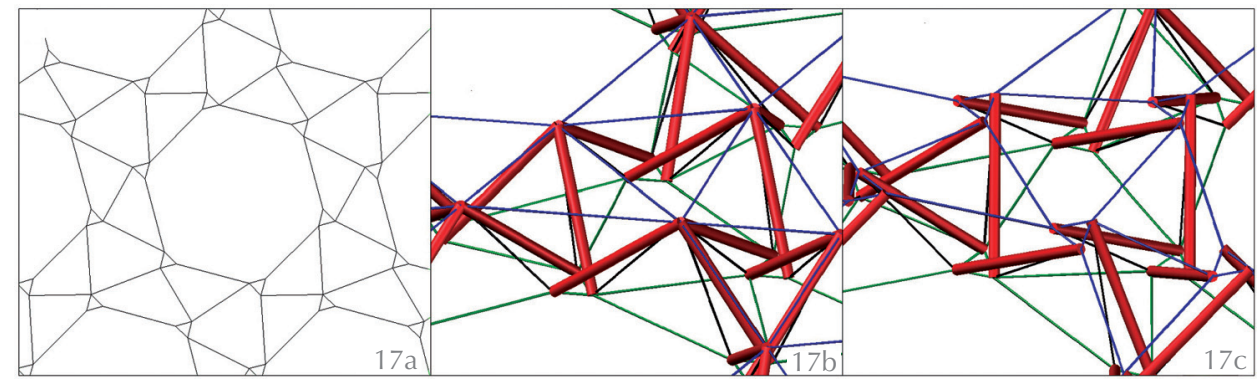

17. MTDC a partir de MDC de mosaico 4,6,12: a) Manipulación de Rot-Umbela (u=3), b) Resultado aplicado a capa inferior, c) Resultado aplicado a ambas capas.

18. Obtención de la MTDC de Kono\&Kunieda's a partir de la malla de Le Ricolais con Manipulaciones de Rot-Umbela.

Tomando como ejemplo la MDC de la Figura 14c, si se aplica una Manipulación de Rot-Umbela de valencia $3(u=3)$ en la capa de abajo y se le aplica un determinado giro, tal y como muestra la Figura $17 \mathrm{a}$, se genera automáticamente una MTDC compuesta por trípodes rígidos $(k=3)$ comprimidos y aislados entre sí, representada en la Figura 17b. Si adicionalmente se manipula también la capa superior siguiendo la misma técnica, se obtiene la malla tensegrítica de la Figura 17c, donde se puede apreciar que todas las barras están aisladas $(k=1)$ e inmersas en una red continua de elementos traccionados. La agrupación de barras, conformando trípodes tensegríticos, es similar a la que ya emplearan Snelson, Emmerich y Kono, como se vio en la Figura 4.

Este importante apunte sirve de catalizador para inferir que podría haber una similitud relevante y reveladora entre estos tipos de mallas y procesos constructivos. Para intentar responder a este interrogante, diferentes mallas convencionales fueron testadas para comprobar si eran capaces de generar mallas tensegríticas conocidas en la actualidad. Como primer hito, se consiguieron resultados satisfactorios a partir de una maIla de Le Ricolais (que se puede generar a su vez siguiendo la metodología de Otero aplicando leyes de alternancia a una malla regular hexagonal, como se ve en la Figura $18 \mathrm{a}$ y $18 \mathrm{~b})$. Aplicando una Rot-Umbela de $\mathrm{u}=3$ con rotación media de $150^{\circ}$ a ambas capas superior e inferior (Figura 18c), el resultado final no es otro que la obtención de la MTDC de Kono \& Kunieda's (Figura 18d) de la cual ya habláramos y reseñáramos en párrafos precedentes (ver Figura 4c).

Siguiendo esta línea de investigación, también se ha podido deducir que existe otro caso especial en el que se pueden obtener derivaciones análogas. Esta vez, la estructura de origen es una de las MDC más empleadas en la arquitectura e ingeniería, la Space Deck, también denominada estructura espacial de semioctaedros, malla de base cuadrada, square-on-square, etc. (Figura 19a). A partir de ella, y aplicándole en la capa superior una Manipulación de Rot-Umbela de valencia natural $4(v=u=4)$ (Figura 19b) sucedida por un giro de $135^{\circ}$

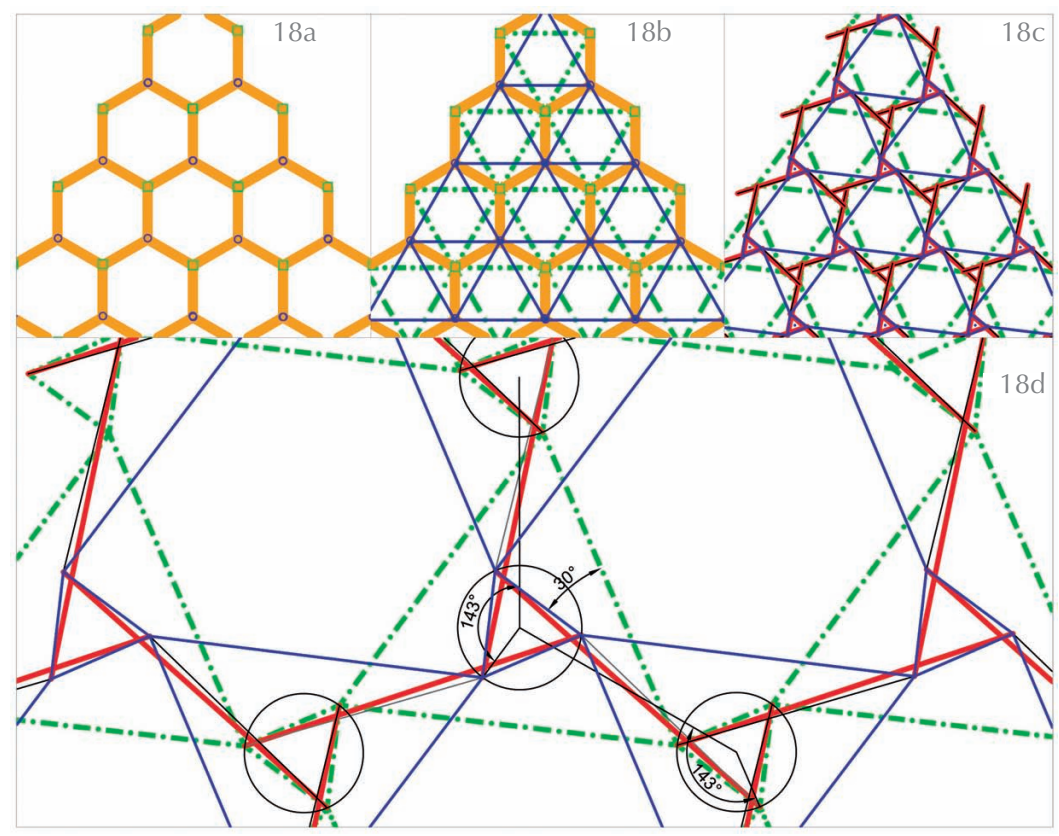

en torno a cada vértice (Figura 19c-e), se obtiene la misma malla tensegrítica de la Figura 3, compuesta por semicuboctaedros tensegríticos contiguos, con la que comenzara su análisis de propuestas René Motro en los años 80.

Como ya se ha mencionado, la geometría final depende de varios parámetros, a saber, radio de apertura de los vértices, ángulo de giro de los nuevos polígonos creados, estado de pretensado de la estructura, etc.

Es comúnmente sabido por parte de los duchos en la materia, que tanto en las tensegridades cilíndricas o prismáticas, como en las piramidales truncadas, existe un ángulo de giro $(\alpha)$ entre ambas bases que depende directa y exclusivamente del número de barras de la estructura $(n)$, siguiendo la fórmula $\alpha=90^{\circ}-180^{\circ} / \mathrm{n}$. En las Manipulaciones de Rot-Umbela, como comentado previamente, también aparece un ángulo de giro dependiente de otros tantos factores, pero que no es el objetivo de este texto desarrollar ahora. Aun así, como en todas las estructuras de compresión flotante, es posible corregir la geometría final variando el estado de pretensado o añadiendo tendones adicionales a la propia estructura. 
19. Obtención de la MTDC de "quadruplex" piramidal a partir de la malla Space Deck con Manipulaciones de Rot-Umbela.

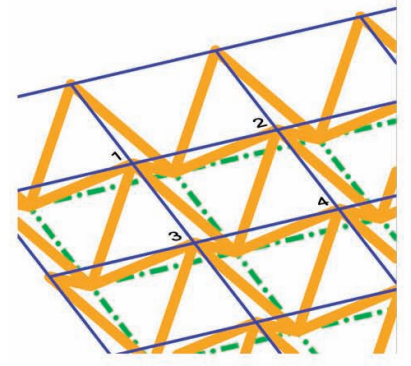

$19 \mathrm{a}$

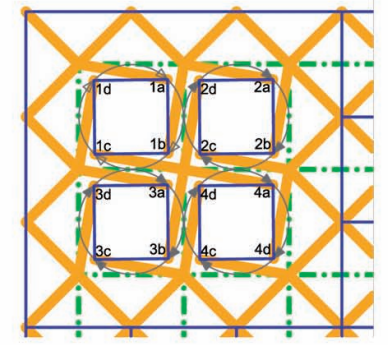

$19 d$

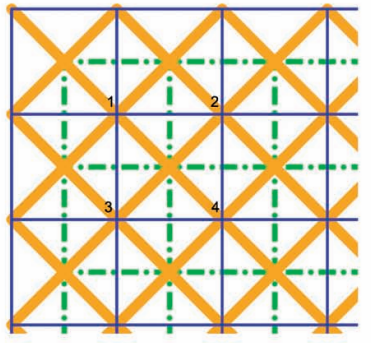

$19 b$

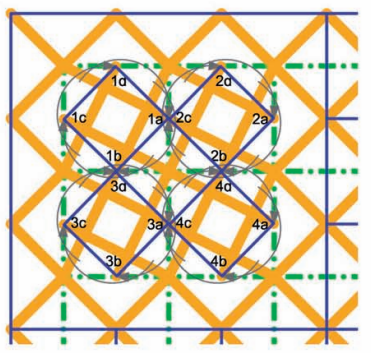

$19 \mathrm{e}$

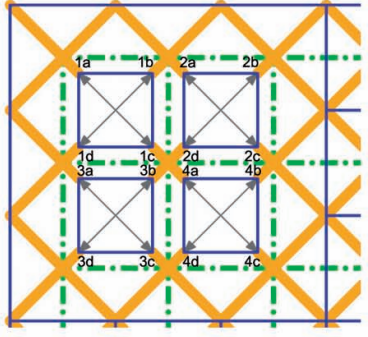

$19 \mathrm{c}$

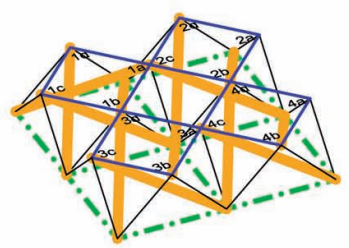

$19 f$

\subsection{Análisis estructural de las mallas}

Con el fin de conseguir configuraciones estables y plausibles de las MTDC en general, y de las presentadas previamente en particular, es esencial probar su estabilidad estructural y su equilibrio por medio de un estudio analítico riguroso del modelo (81) o, como vía adicional o alternativa, mediante la construcción correcta de una maqueta o modelo de dicho ejemplar. Siendo este el caso, durante la presente investigación se han Ilevado a cabo ambas alternativas, contando la primera con el empleo de procesos numéricos basados inicialmente en el método de densidad de fuerzas (82), algoritmos de relajación dinámica modificada (83), y más adelante con herramientas específicas desarrolladas para el análisis de estructuras espaciales reticulares ligeras, como son las tensegridades, más concretamente mediante el software ToyGL (84). Existe abundante bibliografía con ejemplos numéricos relativos al primer método, por lo que no ahondaremos en el tema para no incrementar innecesariamente la extensión de esta exposición.

De todos modos, huelga decir que siempre se habrá de prestar especial atención y detallado cuidado a la geometría del contorno de las mallas tensegríticas, pues su disposición es crítica a la hora de proveer de equilibrio estable al sistema en su conjunto.

\section{APLICACIONES}

No cabe duda que estando enmarcados en un contexto arquitectónico e ingenieril, es necesario ir más allá de las propias funcionalidades que ostentan per se las Manipulaciones de Rot-Umbela; estas son esencialmente el descubrimiento de nuevas configuraciones de estructuras espaciales planas tensegríticas, y la generación de las ya existentes de modos alternativos.

Una vez superado el plano teórico, es imprescindible darle una aplicación concreta a este tipo de estructuras para poder justificar los esfuerzos que tantos especialistas están invirtiendo en ellas. Aunque en este apartado no se entre en detalle en este aspecto, sí que es conveniente, por lo menos, esbozar levemente algunas propuestas que se están planteando a este respecto.

Diversos estudios de arquitectura $(A B D R$, Lift Architects, Orambra, Blue Office Architecture, etc.) están incorporando en sus propuestas, con más y más frecuencia, MTDC para la cubrición de espacios públicos, desde pequeñas marquesinas, hasta tejados extensos integrados en centros de exposición, bibliotecas, museos, estaciones de tren, etc. También hay proyectos en los que estructuras tensegríticas planas se habilitan como paredes técnicas o muros de separación sin requerimientos de función portante. Algunas "pseudo-tensegridades", como ya se expuso en la introducción, ya son realidad en cubiertas de estadios deportivos, llegando a distancias que baten records de luz salvada. Y aunque hay otros planteamientos que no son MTDC propiamente dichas, también se están haciendo propuestas para ser aplicadas a líneas eléctricas de media y alta tensión (85).

\section{PROPUESTAS DE ANÁLISIS POR TRATAR}

Decía Ramón y Cajal que no hay cuestiones agotadas sino hombres agotados en las cuestiones. Afortunadamente, el campo de estudio de la Tensegridad es amplísimo y de implicaciones notorias, por lo que no hace 
falta ir a buscar preguntas adicionales, éstas acuden a uno directamente sin Ilamarlas. Por citar sólo algunos de los temas que se estudian y/o estudiarán en línea con esta investigación, merecen especial atención la comparación analítica del comportamiento estructural, eficiencia material, económica y constructiva entre las MDC y las MTDC, tras la "tensegrización" de éstas, o bien en función de sus luces, masas o capacidades portantes similares de unas y otras. Es obvio que las principales ventajas o inconvenientes de ambas tipologías entrarán en liza dependiendo de la aplicación final que se les dé. Por lo tanto, entre los objetivos prioritarios estará el de encontrar funcionalidades eficientes y propias a las MTDC, que hasta el momento no se están hallando de manera totalmente satisfactoria.

\subsection{Otras líneas de investigación}

Como añadido a todo lo expuesto en secciones precedentes, se están llevando a cabo paralelamente otras vías de estudio para la búsqueda de nuevas configuraciones de MTDC, que serán expuestas en futuros trabajos, de forma más extensa y detallada. Como apunte, decir que algunas de ellas nacen de la variación de MDC convencionales, otras surgen directamente de la combinación de mosaicos tradicionales que conforman las distintas capas de las mallas tensegríticas a obtener, mientras que otras por ejemplo aparecen como superposiciones de MTDC ya existentes.

En cuanto a aplicaciones, no se descarta el intento de análisis de MTDC como pasarelas de un solo vano, en las que algunos de sus cables sean sustituidos por membranas, de modo similar a otras experiencias que se están Ilevando a cabo en nuestro país (86).

\section{CONCLUSIONES}

Aunque durante los últimos años se han realizado diversas propuestas para diseñar
MTDC, la mayoría de ellas se han basado en una metodología de composición, es decir, consistente en agrupar módulos tensegríticos básicos, ya de por sí estables de manera independiente. Tal y como demostró Raducanu, existe un camino importante por recorrer a lo largo de las diferentes variantes que se pueden obtener mediante metodologías de descomposición.

En este artículo se ha realizado una nueva propuesta basada en Manipulaciones de Rot-Umbela, tanto para generar nuevas mallas de compresión flotante, como para demostrar que dos de las más importantes y empleadas durante las últimas décadas son también el resultado de aplicar este método a MDC convencionales. Este hecho abriga la idea de que nuevas e interesantes configuraciones se pueden obtener eligiendo correctamente la malla espacial de origen y la Rot-Umbela adecuada, abriendo un sinfín de posibilidades a un catálogo de MTDC que está lejos de agotarse.

\section{AGRADECIMIENTOS}

El autor querría agradecer la desinteresada y generosa colaboración de Julien Aversen y Landolf Rhode-Barbarigos, poniendo a disposición para esta investigación herramientas de gran utilidad para el análisis de las estructuras aquí expuestas, como son respectivamente el ToyGL (84) y el algoritmo de relajación dinámica modificada (83).

Asimismo, también se quiere mencionar la generosa participación de la Demarcación del País Vasco del Colegio de Ingenieros de Caminos Canales y Puertos, que ha apoyado este proyecto de investigación durante buena parte del tiempo en el que se ha llevado a cabo. Esta colaboración desinteresada es fruto de la apuesta de esta institución por el progreso, avance y desarrollo de la ingeniería, fundamentado todo ello en los pilares de la educación y la investigación.

\section{BIBLIOGRAFÍA}

(1) Gómez-Jáuregui, V.: Tensegrity structures and their application to architecture, Santander: Universidad de Cantabria. Servicio de Publicaciones, 2010.

(2) Cuevas Riaño, M.: "Estructuras lógicas en las artes plásticas", Tesis Doctoral, Universidad Complutense de Madrid, 2007.

(3) Gómez-Jáuregui, V.: "Controversial Origins of Tensegrity", en Symposium of the International Association for Shell and Spatial Structures (50th. 2009. Valencia). Evolution and Trends in Design, Analysis and Construction of Shell and Spatial Structures: Proceedings/Alberto Domingo and Carlos Lázaro, eds., 2009.

(4) Fuller, B. R.: "Tensile-integrity structures", Patente US3063521, concedida 13-Oct-1962.

(5) Snelson, K. D.: "Continuous tension, discontinuous compression structures", Patente US3169611, concedida 16-Feb-1965.

(6) Emmerich, D. G.: "Construction de réseaux autotendants", Patente FP1377290, concedida 28-Sep-1964. 
(7) Emmerich, D. G.: "Structures linéaires autotendants", Patente FP1377291, concedida 28-Sep-1964.

(8) Burkhardt, R.: "Snelson's Planar Pieces". [Online]. Disponible en: http://www.trip. net/ bobwb/ts/synergetics/photos/planar.html. [Acceso: 26-Jan-2011].

(9) Snelson, K.: Correspondencia personal con el autor, 16-Oct-2010.

(10) Pugh, A.: "An introduction to tensegrity", Berkeley: University of California Press, 1976.

(11) Vilnay, O.: "Structures made of infinite regular tensegric nets", Bulletin of the International Association of Shell and Spatial Structures, vol. 18, no. 63, pp. 51-57, 1977.

(12) Hanaor, A.: "Preliminary investigation of double-layer tensegrities", en Proceedings of International Conference on the Design and Construction of Non-conventional Structures, vol. 2, 1987.

(13) Hanaor, A.; Liao, M.-K.: "Double-layer tensegrity grids: static load response. I: analytical study", Journal of structural engineering, vol. 117, no. 6, pp. 1660-1674, 1991.

(14) Hanaor, A.: "Double-layer tensegrity grids: static load response. II: experimental study", Journal of structural engineering, vol. 117, no. 6, pp. 1675-1684, 1991.

(15) Hanaor, A.: "Aspects of design of double-layer tensegrity domes", International Journal of Space Structures, vol. 7, no. 2, pp. 101-113, 1992.

(16) Hanaor, A.: "Double-layer tensegrity grids as deployable structures", International Journal of Space Structures, vol. 8, no. 1-2, pp. 135-143, 1993.

(17) Hanaor, A.: "Geometrically rigid double-layer tensegrity grids", International Journal of Space Structures, vol. 9, no. 4, pp. 227-238, 1994.

(18) Hanaor, A.: "Tensegrity: Theory and application", en Beyond the Cube: The Architecture of Space Frames and Polyhedra, J. Francois Gabriel, John Wiley and Sons, Ic, pp. 385-408, 1997.

(19) Motro, R.: "Tensegrity systems for double layer space structures", en Proceedings of International Conference on the Design and Construction of Non-conventional Structures, vol. 2, 1987.

(20) Motro, R.: "Tensegrity systems: the state of the art", International Journal of Space Structures, vol. 7, no. 2, pp. 75-83, 1992.

(21) Motro, R.: "Tensegrity systems : latest developments and perspectives", presentado en 10 years of progress in Shell and Spatial Structures. IASS Symposium 1989, Madrid, 1989.

(22) Motro, R.: Tensegrity: Structural systems for the future, London, Sterling, V. A: Kogan Page Science, 2003.

(23) Motro, R.: "Structural morphology of tensegrity systems", Asian Journal of Civil Engineering, vol. 10, no. 1, pp. 1-19, 2009.

(24) Maurin, B.; Motro, R.; Raducanu, V.; Pauli, N.: "Soft 'tensegrity like' panel: Conceptual design and Form - Finding", Journal of the International Association for Shell and Spatial Structures, vol. 49, no. 158, pp. 77-87, 2008.

(25) Nestorovic, M.: "Metallic Integrally Tensioned (Tensegrity) Cupola", en Proceedings of International Conference on the Design and Construction of Non-conventional Structures, vol. 2, 1987.

(26) Nestorovic, M.: "Analysis of the properties of integrally tensioned tensegrity domes", presentado en Procedings of the $4^{\text {th }}$ International Conference on Space Structures, London, 1993.

(27) Emmerich, D. G.: Structures tendues et autotendantes, École d'architecture de Paris la Villette, 1988.

(28) Emmerich, D. G.: "Self-tensioning spherical structures: single and double layer spheroids", International Journal of Space Structures, vol. 5, no. 3, pp. 335-374, 1990.

(29) Emmerich, D. G.: "Emmerich on self-tensioning structures", International Journal of Space Structures, vol. 11, no. 1-2, pp. 29-36, 1996.

(30) Wang, B.-B.; Liu, X.-L.: "Integral-tension research in double-layer tensegrity grids", International Journal of Space Structures, vol. 11, no. 4, pp. 349-355, 1996.

(31) Wang, B.-B.: "Simplexes in tensegrity systems", Journal of the International Association for Shell and Spatial Structures, vol. 40, no. 129, pp. 57-64, 1999.

(32) Wang, B.-B.: "Cable-Strut Systems: Part II - Cable-Strut", Journal of Constructional Steel Research, vol. 45, no. 3, pp. 291-299, 1998.

(33) Wang, B.-B.: "Cable-Strut Systems: Part I - Tensegrity", Journal of Constructional Steel Research, vol. 45, no. 3, pp. 281-289, 1998.

(34) Wang, B..-B.; Li, Y. Y.: "RP system-properties, design and applications", en Proceedings Of The International Symposium of Lightweight Structures In Civil Engineering, 2002.

(35) Wang, B.; Li, Y.: "Novel cable-strut grids made of prisms: Part I. Basic theory and design", Journal of the International Association for Shell and Spatial Structures, vol. 44, no. 142, pp. 93-108, 2003.

(36) Wang, B.; Li, Y.: "Novel cable-strut grids made of prisms: Part II. Deployable and architectural studies", Journal of the International Association for Shell and Spatial Structures, vol. 44, no. 142, pp. 109-125, 2003. 
(37) Wang, B.: Free-standing tension structures: from tensegrity systems to cable-strut systems, London; New York: Spon Press, 2004.

(38) Wang, B.B.; Li, Y.Y.: "Cable-strut systems of non-contiguous strut configurations - Morphological study", Journal of the International Association for Shell and Spatial Structures, vol. 46, no. 147, pp. 23-39, 2005.

(39) Hilyard, M. J.; Lalvani, H.: "Emmerich-type structures constructed fromtensegrity modules", presentado en IASS Structural Morphology Colloquium 2000, Bridge Between Civil Engineering \& Architecture, Delft, p. 196-205, 2000.

(40) Kono, Y.; Kunieda, H.: "Tensegrity grids transformed from double-layer space grids", presentado en Proceedings Conceptual Design of Structures, IASS, Stuttgart, pp. 293300, 1996.

(41) Kono, Y.; Kunieda, H.: "A class of double-layer tensegrity grid domes", Singapore, pp. 455-463, 1997.

(42) Kono, Y.; Kunieda, H.: "Experimental Study On Static Load Responses Of Double Layer Tensegrity Grids", J. Struct. Constr. Eng., AlJ, no. 502, pp. 93-97, 1997.

(43) Kono, Y.; Kunieda, H.; Shimada, T.: "Form-Finding Of Double-Layer Tensegrity Grids With Multiple States of Self-Stress", J. Struct. Constr. Eng., AlJ, no. 501, pp. 73-76, 1997.

(44) Kono, Y.; Choong, K.K.; Shimada, T.; Kunieda, H.: "An experimental investigation of a type of double-layer tensegrity grids", Journal of the International Association for Shell and Spatial Structures, vol. 40, no. 130, pp. 103-111, 1999.

(45) Kono, Y.; Kunieda, H.: "Frame Structure And Method For Forming The Same", Patente WO9744543, concedida 27-Feb-2001.

(46) Quirant, J.; Motro, R.; Kazi-Aoual, M.: "Designing tensegrity systems: the case of a double layer grid", Engineering Structures, vol. 25, no. 9, pp. 1121-1130, DOI: 10.1016/S01410296(03)00021-X, 2003.

(47) Quirant, J.: "Systèmes de tenségrité et autocontrainte: Qualification, sensibilité et incidence sur le comportement", Tesis Doctoral, Université de Montpellier II, 2000.

(48) Quirant, J.; Kazi-Aoual, M. N.; Laporte, R.: "Tensegrity systems: The application of linear programmation in search of compatible selfstress states", Journal of the International Association for Shell and Spatial Structures, vol. 44, no. 141, pp. 33-50, 2003.

(49) Smaili, A.; Motro, R.: "Foldable / unfoldable curved tensegrity systems by finite mechanism activation", Journal of the International Association for Shell and Spatial Structures, vol. 48, no. 155, pp. 153-160, 2007.

(50) Smaili, A.; Motro, R.: "A self-stress maintening folding tensegrity system by finite mechanism activation", Journal of the International Association for Shell and Spatial Structures, vol. 46, no. 148, pp. 85-93, 2005.

(51) Smaili, A.; Motro, R.: "Folding/unfolding of tensegrity systems by removal of self-stress", en International symposium on shell and spatial structures, pp. 595-602, 2005.

(52) Smaili, A.: "Systemes legers pliables/depliables: cas des systemes de tensegrite", Tesis Doctoral, Université de Montpellier II, 2004.

(53) Sánchez, R.; Kazi Aoual, M. N.; Motro, R.: "Pre-Sizing Of Tensegrity Systems", en Proceedings Of The International Symposium, 2002.

(54) Sánchez, R.; Maurin, B.; Kazi-Aoual, M. N.; Motro, R.: "Modélisation des systèmes de tenségrité: Grilles à double nappe", Montpellier: Laboratoire de Mécanique et Génie Civil. Université Montpellier II. Disponible en http://www. lirmm. fr/doctiss04/art/ M, 2003.

(55) Bouderbala, M.; Motro, R.: "Folding tensegrity systems", Solid Mechanics and its applications, vol. 80, pp. 27-36, 2000.

(56) Vassart, N.: "Recherche de forme et stabilité des systèmes réticulés autocontraints Application aux systèmes de tenségrité", Tesis Doctoral, Université de Montpellier II, 1997.

(57) Averseng, J.; Dubé, J.-F.; Crosnier, B.; Motro, R.: "Active control of a tensegrity plane grid," en Proceedings of the $44^{\text {th }}$ IEEE Conference on Decision and Control, and the European Control Conference, CDC-ECC '05, vol. 2005, pp. 6830-6834, 2005.

(58) Averseng, J.: "Mise en oeuvre et contrôle des systèmes de tenségrité", Tesis Doctoral, Université de Montpellier II, 2004.

(59) Averseng, J.; Crosnier, B.: "Static and dynamic robust control of tensegrity systems", Journal of the International Association for Shell and Spatial Structures, vol. 45, no. 146, pp. 169-174, 2004.

(60) Averseng, J.; Crosnier, B.: "Prestressing tensegrity systems - Application to multiple selfstress state structures", International Journal of Structural Stability and Dynamics, vol. 4, no. 4, pp. 543-557, 2004.

(61) Djouadi, S.; Motro, R.; Pons, J. C.; Crosnier, B.: "Active control of tensegrity systems", Journal of Aerospace Engineering, vol. 11, no. 2, pp. 37-43, 1998. 
(62) Kebiche, K.: "Etude en non-linéarités géométriques et homogénéisation des systèmes réticulés autocontraints : application aux systèmes de tenségrité", Tesis Doctoral, Université de Montpellier II, 1998.

(63) Le Saux, C.: "Modélisation numérique du pliage et du déploiement des systèmes spatiaux avec prise en compte des contacts et des frottements", Tesis Doctoral, Université de Montpellier II, 2002.

(64) Raducanu, V.: "Architecture Et Système Constructif - Case De Systémes De Tenségrité", Tesis Doctoral, Université de Montpellier II, 2001.

(65) Pedretti, M.: "Smart tensegrity structures for the Swiss Expo 2001", en Proceedings of SPIE - The International Society for Optical Engineering, vol. 3330, pp. 378-386, 1998.

(66) Adriaenssens, S. M. L.; Barnes, M. R.: "Tensegrity spline beam and grid shell structures", Engineering Structures, vol. 23, no. 1, pp. 29-36. DOI: 10.1016/S0141-0296(00)000195, 2001.

(67) Panigrahi, R.; Gupta, A.; Bhalla, S.: "Design of tensegrity structures using artificial neural networks", Structural Engineering and Mechanics, vol. 29, no. 2, pp. 223-235, 2008.

(68) Panigrahi, R.; Gupta, A.; Bhalla, S.: "Dismountable steel tensegrity grids as alternate roof structures", Steel and Composite Structures, vol. 9, no. 3, pp. 239-253, 2009.

(69) Tran, H. C.; Lee, J.: "Advanced form-finding of tensegrity structures", Computers \& Structures, vol. 88, no. 3-4, pp. 237-246, DOI: 10.1016/j.compstruc.2009.10.006, 2010.

(70) Tran, H. C.; Lee, J.: "Initial self-stress design of tensegrity grid structures", Computers and Structures, vol. 88, no. 9-10, pp. 558-566, 2010.

(71) Tran, H. C.; Lee, J.: "Self-stress design of tensegrity grid structures with exostresses", International Journal of Solids and Structures, vol. 47, no. 20, pp. 2660-2671. DOI: 10.1016/j.ijsolstr.2010.05.020, 2010.

(72) Liapi, K.; Kim, J.: "Double-layer tensegrity grids for architectural applications", Structures \& Architecture, P. Cruz, Ed. CRC Press, pp. 429-430, 2010.

(73) Skelton, R.; De Oliveira, M. C.: Tensegrity systems, Dordrecht, London: Springer, 2009.

(74) Skelton, R.; Helton, W. J.; Adhikari, R.: "Mechanics of Tensegrity Beams", UCSD, Structural Systems \& Contr. Lab., Rep. N. ${ }^{\circ} 1998-1,1998$.

(75) Otero, C.: Diseño geométrico de cúpulas no esféricas aproximadas por mallas triangulares con un número mínimo de longitudes de barra, Tesis Doctoral. Universidad de Cantabria, 1990.

(76) Otero, C.; Oti, J.; Villar, F.; Otero, F.: "Classical Geometry in flat and simple curved meshes", Bulletin of the International Association of Shell and Spatial Structures, vol. 33, n 108, pp. 3-31, 1992.

(77) Gómez-Jáuregui, V.; Otero, C.; Arias, R.; Manchado, C.: "Generation and nomenclature of tessellations and double-layer grids", Journal of Structural Engineering-ASCE, July, 2012. DOI: 10.1061/(ASCE)ST.1943-541X.000053

(78) Gómez-Jáuregui, V.; Arias, R.; Otero, C.; Manchado, C.: "Novel technique for obtaining double-layer tensegrity grids", International Journal of Space Structures, vol. 27, número especial 2-3, pp. 155-166, DOI: 10.1260/0266-3511.27.2-3.155.

(79) Gancedo Lamadrid, E.: Estudio de propiedades métricas de las radiaciones centrales en poliedros convexos, Tesis Doctoral, Universidad de Cantabria, 1988.

(80) Gancedo Lamadrid, E.; Álvarez Gómez, J. M; Suárez González, J.; Vega Menéndez, J.: "A New Method to Obtain and Define Regular Polyhedra", Geometriae Dedicata, vol. 106, no 1 (2004), pp. 43-49, DOI: 10.1023/B:GEOM.0000033841.77105.bb.

(81) Tibert, A. G.; Pellegrino, S.: "Review of form-finding methods for tensegrity structures". International Journal of Space Structures, vol. 18, nº (2003), pp. 209-223.

(82) Zhang, J.Y.; Ohsaki, M.: "Adaptive force density method for form-finding problem of tensegrity structures", International Journal of Solids and Structures, vol. 43, n 18-19 (2006), pp. 5658-5673, DOI: 10.1016/j.ijsolstr.2005.10.011.

(83) Bel Hadj Ali, N.; Rhode-Barbarigos, L.; Smith, I. F. C.: "Analysis of clustered tensegrity structures using a modified dynamic relaxation algorithm".

(84) Averseng, J.; Jérome Quirant; Jean-Francois Dubé: "Interactive Design And Dynamic Analysis Of Tensegrity Systems", presentado en Structural Engineers World Congress 2011, Como (Italy), 2011.

(85) Tibert, G.: “Design And Form-Finding Analysis Of Tensegrity Power Lines", presentado en Structural Enginners World Congress 2011, Como (Italy), 2011.

(86) Murcia, J.: "Tecnología de pasarelas con estructura de membrana", Informes de la Construcción, vol. 59, nº 507 (2007), pp. 21-31. 